\title{
Significantly Elevated Liver Alkaline Phosphatase in Congestive Heart Failure
}

\author{
Leonid Shamban ${ }^{\mathrm{a}, \mathrm{b}}$, Brijesh Patel ${ }^{\mathrm{a}}$, Michael Williams ${ }^{\mathrm{a}}$
}

\begin{abstract}
Congestive hepatopathy can have a mildly elevated liver profile, which should normalize with appropriate therapy. Liver specific alkaline phosphatase (ALP) in decompensated heart failure (HF) can be mildly elevated. The levels exceeding beyond the expected rise should be a concern and lead to further investigation. The literature reports insubstantial number of cases regarding significantly elevated levels of ALP and congestive hepatopathy. We report a case of a 45-year-old female with known history of severe cardiomyopathy that had persistently elevated levels of ALP. The extensive workup was negative for any specific pathology. The liver biopsy was consistent with congestive hepatopathy. The patient's ALP levels decreased with aggressive diuretic therapy but still remained elevated.
\end{abstract}

Keywords: Alkaline phosphatase; Congestive hepatopathy; Heart failure

\section{Introduction}

Alkaline phosphatase (ALP) is commonly monitored in clinical practice and can be elevated in various hepatobilliary disease processes. ALP is a heterogenous group of enzymes that catalyze phosphate esters into an organic compound and inorganic phosphate. In humans, it is found in liver, bones, intestine, placenta, kidneys and leukocytes [1]. The liver and bone isoenzymes are the most clinically relevant due to the disproportionate synthesis from these organs. Hepatic ALP is concentrated near the biliary canalicular membrane of the hepatocyte [2], and in pathologic conditions, the respective

Manuscript accepted for publication March 3, 2014

${ }^{a}$ Department of Internal Medicine, Providence Hospital and Medical Center, 16001 W Nile Mile Road, Southfield, MI 48075, USA

${ }^{\mathrm{b}}$ Corresponding author: Leonid Shamban, Department of Internal Medicine, Providence Hospital and Medical Center, 16001 W Nile Mile

Road, Southfield, MI 48075, USA. Email: 1shamban@gmail.com

doi: http://dx.doi.org/10.14740/gr600w isozymes can be elevated which helps to localize the source of the disease. Concomitant elevations of alanine aminotransferase (ALT), aspartate aminotransferase (AST) and gamma-glutamyltransferase (GGT) indicate the elevation of ALP is from a hepatic source [2]. The common causes of elevated ALP include primary sclerosing cholangitis, primary billiary cirrhosis, billiary obstruction, infiltrative diseases, Budd-Chiari obstruction and congestive hepatopathy [3]. In most cases, the ALP levels are mild or moderately elevated in these conditions. The persistently elevated levels of ALP are not only perplexing but often lead to further workup in order to determine the source. Typically very high levels of liver ALP are observed in patients with neoplasms of the liver or billiary tract, severe sepsis, Paget's disease and AIDS $[4,5]$, but reports on high level of ALP in association with heart failure (HF) are lacking. Herein, we report a case of an elevated level of ALP that is out of proportion for a patient with acute on chronic systolic HF.

\section{Case Report}

A 45-year-old African American female with history of severe non-ischemic cardiomyopathy with an ejection fraction of $20 \%$ and poorly controlled diabetes mellitus type 2 , presented to the hospital with difficulty in breathing that had been ongoing for several days. She also complained of orthopnea, lower extremity swelling and a weight gain of least $30 \mathrm{lb}$. Her initial heart rate was 102 beats per minute, blood pressure of 93/73 mm Hg (with her baseline systolic blood pressure, $90-100 \mathrm{~mm} \mathrm{Hg}$ ), respiratory rate of 18 breaths per minute and a temperature of $97.4^{\circ} \mathrm{F}$. The physical exam revealed bibasilar crackles, a regular rate and rhythm, loud P2, jugular venous distention, moderate abdominal distension with significant subcutaneous swelling and $a+2$ pitting edema of the lower extremities.

Her laboratory workup showed NT-proBNP 11,184 pg/ $\mathrm{mL}(192 \mathrm{pg} / \mathrm{mL}$ upper limit of normal), troponin $<0.02 \mathrm{ng} /$ $\mathrm{dL}$ (normal range: $0-0.10 \mathrm{ng} / \mathrm{dL}$ ) and no electrolyte abnormalities. The electrocardiogram did not indicate any signs of ischemia or arrhythmia. Additional laboratory workup revealed AST 28 unit/L, ALT 21 unit/L, ALP 658 unit/L (129 
Table 1. Initial Laboratory Studies the Patient Presented With on Admission

\begin{tabular}{lll} 
Labs on admission & Patient's value & Normal value \\
\hline Prothromin time (PT) & 14.2 & $10.0-13.5 \mathrm{~s}$ \\
INR & 1.2 & $<1.5$ \\
Activated partial thromboplastin time (aPTT) & 31.6 & $25.0-36.0 \mathrm{~s}$ \\
Aspartate aminotransferase (AST) & 28 & $10-35 \mathrm{units} / \mathrm{L}$ \\
Alanine aminotransferase (ALT) & 21 & $10-35 \mathrm{units} / \mathrm{L}$ \\
Albumin & 2.2 & $3.5-5.2 \mathrm{~g} / \mathrm{dL}$ \\
Alkaline phosphatase & 658 & $35-129 \mathrm{units} / \mathrm{L}$ \\
Liver alkaline phosphatase & 95 & $44-84 \%$ \\
Bilirubin total (Bili T) & 1 & $0.1-1.0 \mathrm{mg} / \mathrm{dL}$ \\
Erythrocyte sedimentation rate (ESR) & 9 & $0-20 \mathrm{~mm} / \mathrm{h}$ \\
pro-BNP & 11,184 & $50-192 \mathrm{pg} / \mathrm{mL}$ \\
Hemoglobin A1C & $>17.4$ & $4.8-5.9 \%$ \\
\hline
\end{tabular}

unit/L upper limit of normal), total bilirubin of $1.0 \mathrm{mg} / \mathrm{dL}$ and an albumin of $2.2 \mathrm{~g} / \mathrm{dL}$ (Table 1 ).

Her coagulation panel and platelets were within normal limits. The transthoracic echocardiography showed severe cardiomyopathy with ejection fraction less than 20\% (Fig. 1 ), and right ventricular, inferior vena caval dilatation. Based on the clinical picture, biventricular HF was diagnosed secondary to medication noncompliance, and the patient was treated with aggressive intravenous diuretics.

To investigate the marked elevation of ALP, initially the patient's medications were reviewed, with no history of over-the-counter or herbal medications to explain such a rise.

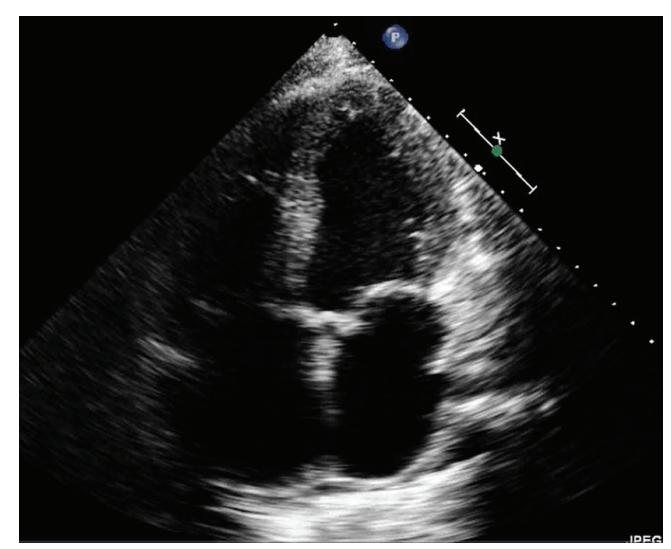

Figure 1. Transthoracic echocardiography (in end-systolic phase) shows an evidence of severe cardiomyopathy with ejection fraction $<20 \%$.
Therefore, an additional workup was then pursued (Table 2). Her ALP continued to trend upwards with mild elevation of transaminases and total bilirubin (Fig. 2).

An ultrasound of the gallbladder revealed calculi with sludge along with wall thickening but no biliary ductal dilatation. A cholescintigraphy scan demonstrated a normal ejection fraction of $45 \%$ with no evidence of cystic duct obstruction. A magnetic resonance cholangiopancreatography (MRCP) revealed pericholecystic fluid with gallbladder wall thickening without evidence of biliary obstruction or suggestive findings of primary sclerosing cholangitis (Fig. 3). A subsequent ultrasound with additive arterial and venous evaluation did not reveal findings of thrombosis.

Despite aggressive therapy, the patient had a positive fluid balance and started to develop signs of cardiorenal syndrome. She was evaluated by an HF specialist, and was deemed not be a candidate for left ventricular assist device or heart transplantation. The patient made an initial response to a furosemide drip with a negative fluid balance, and 48 $\mathrm{h}$ afterwards the patient was switched to oral loop diuretics. However, the subsequent day, the patient continued to retain fluid and ultrafiltration along with hemodialysis was considered. Her ALP continued to rise even with aggressive diuresis and stabilized at 948 unit/L with mild elevation of transaminases with AST 52 unit/L and ALT 40 unit/L. Her total bilirubin slightly increased to $2.2 \mathrm{mg} / \mathrm{dL}$ along with a mild increase in her prothrombin time to $15.5 \mathrm{~s}$ and INR of 1.3.

A CT guided random liver core biopsy was performed 
Table 2. Serological Studies That Were Checked to Evaluate the Elevated ALP

\begin{tabular}{|c|c|c|}
\hline Serological evaluation of ALP & Patient's value & Normal value \\
\hline Thyroid stimulating hormone (TSH) & 2.42 & $0.27-4.20 \mathrm{mcIU} / \mathrm{mL}$ \\
\hline Iron level & 52 & $37-145 \mathrm{mcg} / \mathrm{dL}$ \\
\hline Total iron binding capacity (TIBC) & 281 & $228-428 \mathrm{mcg} / \mathrm{dL}$ \\
\hline Iron saturation & 19 & $20-55 \%$ \\
\hline Ferritin & 47 & $13-150 \mathrm{ng} / \mathrm{mL}$ \\
\hline Ceruloplasmin & 43 & $16-45 \mathrm{mg} / \mathrm{dL}$ \\
\hline Anti-nuclear antibody & Negative & Negative \\
\hline Mitochondrial antibody & Nondetected & Nondetected \\
\hline Smooth muscle antibody & Nondetected & Nondetected \\
\hline Hepatitis A IgM & Nondetected & Nondetected \\
\hline Hepatitis B core antibody IgM & Nondetected & Nondetected \\
\hline Hepatitis B surface antigen & Nondetected & Nondetected \\
\hline Hepatitis $\mathrm{C}$ antibody & Nondetected & Nondetected \\
\hline Angiotensin converting enzyme & 85 & $8-52$ units/L \\
\hline Alfa fetoprotein & 1.1 & $0-13.0 \mathrm{ng} / \mathrm{mL}$ \\
\hline Carcinoembryonic antigen (CEA) & 4.7 & $<5.0 \mathrm{ng} / \mathrm{mL}$ \\
\hline Cancer antigen 19-9 (CA 19-9) & 2 & $0.0-35.0$ units $/ \mathrm{mL}$ \\
\hline Cytomegalovirus (CMV) antibody IgM & 0.03 & $0-0.69$ index \\
\hline Cytomegalovirus (CMV) antibody IgG & 0.2 & $0.0-0.8 \mathrm{AI}$ \\
\hline Herpes simplex virus (HSV) 1 antibody IgM & 0.78 & $0.00-0.90$ index \\
\hline Herpes simplex virus (HSV) 1 antibody IgG & 4.4 & $0.0-0.8 \mathrm{AI}$ \\
\hline Quantiferon TB-gold & Negative & Negative \\
\hline
\end{tabular}

with four specimens obtained. The liver biopsy findings revealed venous congestion and mild biliary obstruction (Fig. 4). There was no evidence to suggest sarcoidosis, amyloidosis or lymphocytic malignancies. Unfortunately, later in the hospitalization the patient went into respiratory failure followed by cardiac arrest.

\section{Discussion}

This case represents an atypical presentation of an elevated liver ALP in the presence of severe congestive HF. Con- gestive hepatopathy can have mildly elevated liver profile, which should normalize with appropriate therapy. Hepatic congestion may respond to diuresis, but in severe HF, augmentation of cardiac output may also be required to prevent further deterioration of liver function. In cases that are refractory, patients may undergo ultrafiltration to remove edema/ascites that is no longer responsive to diuretic therapy [6]. This notion is seen in our patient whose ALP started to improve when there was response to diuresis therapy (Fig. 2 ). However, as the diuresis waned ultrafiltration and hemodialysis was then considered.

Specific ALP in decompensated HF can be mildly ele- 


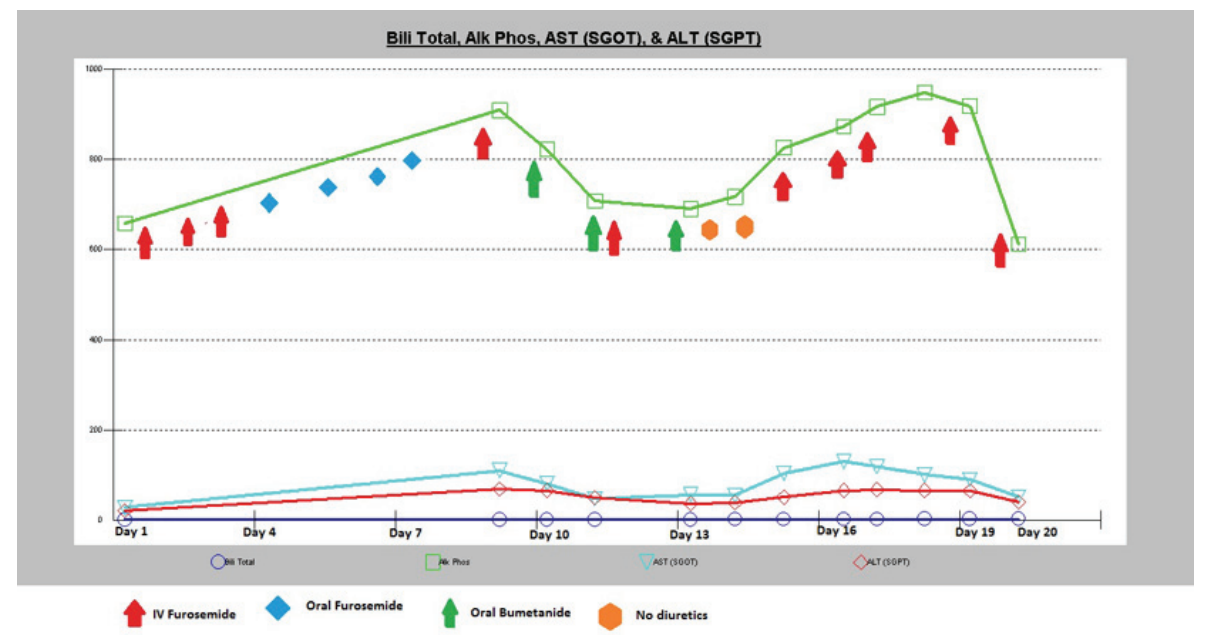

Figure 2. This graph illustrates how ALP levels responded to various types of diuretic therapies along with the other liver enzymes progressions; total bilirubin, AST and ALT levels.

vated. Abnormalities in liver enzymes are not an uncommon finding in patients with HF [6]. Increased levels of AST and ALT in HF have been attributed to hepatocellular damage from decreased perfusion, whereas high levels of ALP and increased bilirubin have been associated with cholestatic liver injury from an increased central venous pressure [7]. Two HF trials performed in recent past, the EVEREST (Efficacy of Vasopressin Antagonism in Heart Failure Outcome Study with Tolvaptan) and the CHARM (Candesartan in Heart Failure Assessment of Reduction in Mortality and Morbidity) trial suggested that minor abnormalities are common in patients hospitalized for worsening HF with reduced ejection fraction without a history of significant primary liver disease or acute hepatic failure [8].

Typically, a liver specific ALP elevation disproportionate to the rise in aminotransferase suggests a cholestatic disorder. A four-fold elevation of the serum alkaline phosphatase is seen in approximately $75 \%$ of patients with chronic cholestasis, both intrahepatic and extrahepatic, whereas lesser elevations are nonspecific, and can occur in a wide range of conditions [9]. In our patient, imaging studies along with biopsy findings correlated with a partial cholestasis picture, but the ALP levels were much higher than expected. To date there is no evidence in the literature that chronic biliary cholestasis with congestive hepatopathy is additive in the ALP levels. There have been a few reports on extremely elevated ALP (greater than 1,000 U/L) in patients with progressive severe HF [10], but these patients typically had worsening renal function on hospital admission.

\section{Conclusion}

A persistently elevated level of ALP often leads to further evaluation. Mild to moderate elevation of ALP in HF patients has been well documented in the literature, but very high levels of ALP are rarely reported. Our patient showed an improvement in the levels of ALP after aggressive diuretic therapy suggesting that the congestive hepatopathy was the most likely cause of highly elevated ALP level. Future clinicians who are managing patients with systolic decom-
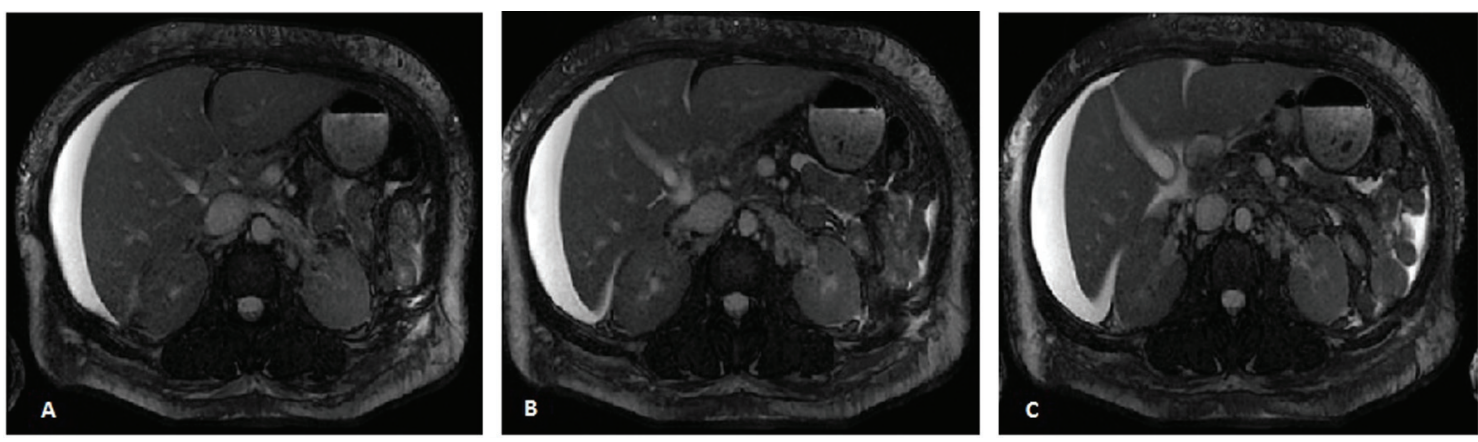

Figure 3. Three consecutive axial MR images, images $A-C$, of the abdomen demonstrating a normal appearing liver with moderate perihepatic ascites along with gallbladder wall thickening. Biliary dilatation is not demonstrated, with a normal pancreatic duct and a common bile duct measuring $3 \mathrm{~mm}$. 

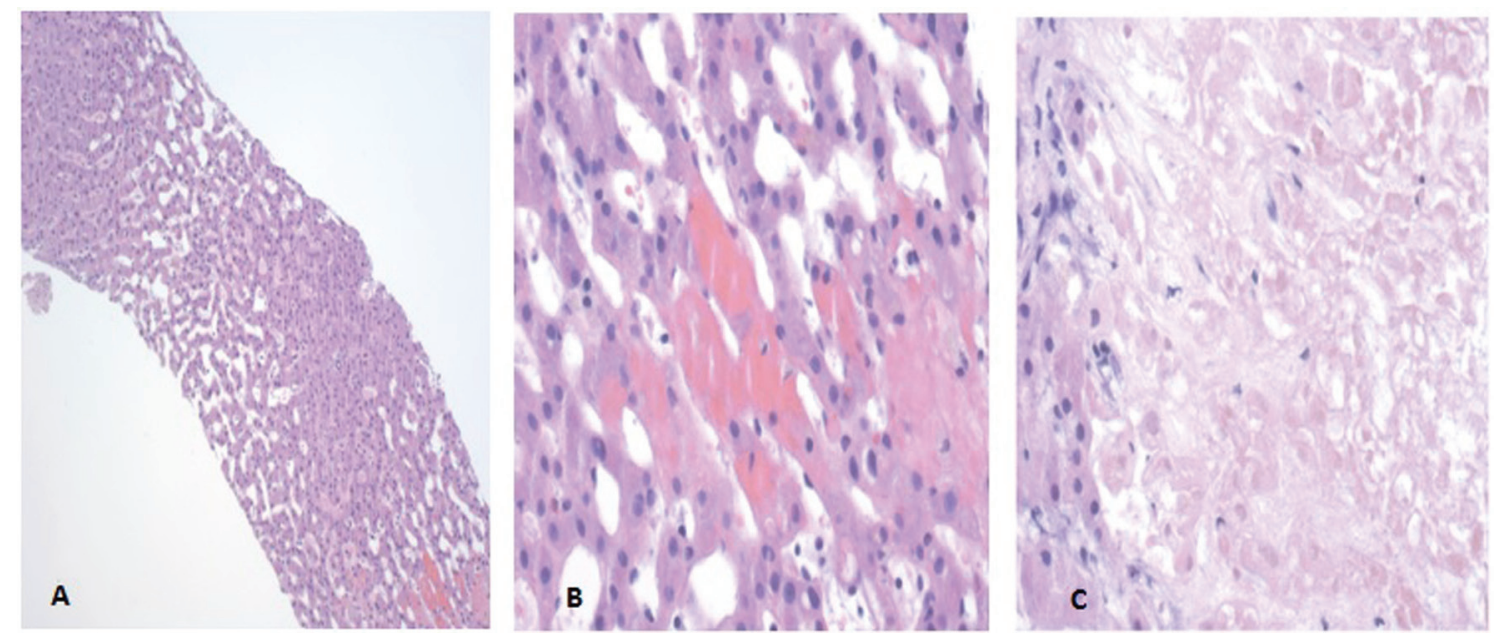

Figure 4. Liver Biopsy Pathology Slides. (A) The hematoxylin and eosin (H\&E) stain at 100x depicting dilated sinusoids of the liver suggestive of right-sided heart failure. (B) The H\&E stain at $400 \times$ depicting erythrocytes in hepatic plates suggestive of leftsided heart failure. (C) The H\&E stain at $400 \times$ demonstrating biliary infarction consistent with mild biliary obstruction.

pensated HF with concurrent biliary sludge and an elevated ALP greater than what is typically expected should consider congestive hepatopathy as the etiologic source. Therefore, a liver biopsy might not be warranted if the clinical suspicion is low for other pathologies, with normal serological studies and appropriate negative imaging studies.

\section{Acknowledgement}

None.

\section{Conflict of Interest}

None.

\section{References}

1. Wiwanitkit V. High serum alkaline phosphatase levels, a study in 181 Thai adult hospitalized patients. BMC Fam Pract. 2001;2:2.

2. Esfeh JM, Hanouneh IA, Zein NN. A 25-year-old man with very high alkaline phosphatase. Cleve Clin J Med. 2011;78(12):793-800.

3. Siddique A, Kowdley KV. Approach to a patient with elevated serum alkaline phosphatase. Clin Liver Dis. 2012;16(2):199-229.
4. Maldonado O, Demasi R, Maldonado Y, Taylor M, Troncale F, Vender R. Extremely high levels of alkaline phosphatase in hospitalized patients. J Clin Gastroenterol. 1998;27(4):342-345.

5. Tung CB, Tung CF, Yang DY, Hu WH, Hung DZ, Peng YC, Chang CS. Extremely high levels of alkaline phosphatase in adult patients as a manifestation of bacteremia. Hepatogastroenterology. 2005;52(65):1347-1350.

6. Samsky MD, Patel CB, DeWald TA, Smith AD, Felker GM, Rogers JG, Hernandez AF. Cardiohepatic interactions in heart failure: an overview and clinical implications. J Am Coll Cardiol. 2013;61(24):2397-2405.

7. van Deursen VM, Damman K, Hillege HL, van Beek AP, van Veldhuisen DJ, Voors AA. Abnormal liver function in relation to hemodynamic profile in heart failure patients. J Card Fail. 2010;16(1):84-90.

8. Ambrosy AP, Vaduganathan M, Huffman MD, Khan S, Kwasny MJ, Fought AJ, Maggioni AP, et al. Clinical course and predictive value of liver function tests in patients hospitalized for worsening heart failure with reduced ejection fraction: an analysis of the EVEREST trial. Eur J Heart Fail. 2012;14(3):302-311.

9. Mark Feldman M, Lawrence S. Friedman, MD and Lawrence J. Brandt, MD. Sleisenger and Fordtran's Gastrointestinal and Liver Disease. 2010;2:1231-1234,1380-1381.

10. Mshe'el S, Bisharat N. Extremely high levels of serum alkaline phosphatase in patients with prolonged hepatic congestion. Eur J Gastroenterol Hepatol. 2011;23(5):444. 